\title{
Characteristic peripheral blood findings in human ehrlichiosis
}

\author{
Katherine S Hamilton ${ }^{1}$, Steven M Standaert ${ }^{2}$ and Marsha C Kinney ${ }^{1}$ \\ ${ }^{1}$ Department of Pathology and ${ }^{2}$ Department of Medicine, Division of Infectious Disease, Vanderbilt University \\ Medical Center, Nashville, TN, USA
}

\begin{abstract}
Human ehrlichiosis is a potentially fatal tick-borne illness if not treated promptly. Ehrlichia infection is difficult to diagnose as the organism does not grow in standard blood culture medium and serological confirmation of infection takes several days to weeks. The most timely way of confirming Ehrlichia infection is identification of characteristic cytoplasmic morulae in peripheral blood leukocytes. A total of 23 patients with clinical and laboratory findings suggesting a rickettsial infection were tested for Ehrlichia using polymerase chain reaction and culture: 16 cases contained Ehrlichia DNA by polymerase chain reaction (15 E. chaffeensis, one E. ewingii), including 14 cases in which the blood culture grew Ehrlichia. The cases that contained Ehrlichia DNA by polymerase chain reaction had lower mean white blood cell and platelet counts and more numerous atypical lymphocytes and pronounced toxic change than cases in which Ehrlichia DNA was not detected. Cytoplasmic morulae were identified on peripheral blood smears in six (five E. chaffeensis, one E. ewingii) of $16(38 \%)$ of the cases that contained Ehrlichia DNA, including $4 / 4(100 \%)$ immunocompromised and 2/12 (17\%) immunocompetent patients. Morulae were present in monocytes in $E$. chaffeensis-infected cases and granulocytes in the E. ewingii-infected case. In two immunocompromised patients, the number of infected cells was 1-10\%, but in four patients it was $<0.2 \%$. In conclusion, peripheral blood film examination is diagnostic in a substantial number of Ehrlichia infections, particularly in immunocompromised patients. The number of infected white blood cells may be less than $0.2 \%$, requiring examination of more than $\mathbf{5 0 0}$ white blood cells. Associated changes prompting careful film review include prominent toxic granulation and atypical large granular lymphocytes. Modern Pathology (2004) 17, 512-517, advance online publication, 20 February 2004; doi:10.1038/modpathol.3800075
\end{abstract}

Keywords: Ehrlichia; ehrlichiosis; peripheral blood; morulae

Ehrlichiosis is a tick-borne disease caused by one of several species of Ehrlichia, a Gram-negative obligate rickettsia-like intracellular coccobacillus. Initially recognized in the early 20th century as a zoonotic infection in ruminants and dogs, human infection by several different species has been described. ${ }^{1-5}$ E. chaffeensis is transmitted by Amblyomma americanum $^{6}$ and primarily infects monocytes and produces human monocytic ehrlichiosis. ${ }^{7}$ Another species similar to E. phagocytophilia and E. equi is transmitted by Ixodes scapularis and infects granulocytes, and produces human granulocytic ehrlichiosis in patients in the midwest and northeast. ${ }^{8,9}$ This organism has been referred to a the 'HGE agent' in the past; however, a recent reorganization of the Ricettsiaceae and Anaplasmataceae families based on genetic analyses of 16S rRNA genes, groESL, and

Correspondence: KS Hamilton, Vanderbilt University Medical Center, Department of Pathology, 4601 The Vanderbilt Clinic, Nashville, TN 372325310, USA.

E-mail: katherine.s.hamilton@mcmail.vanderbilt.edu

Received 7 November 2002; revised 17 October 2003; accepted 11 November 2003; published online 20 February 2004 surface protein genes includes the previous 'HGE agent' in the Anaplasmataceae family, now called Anaplasma phagocytophila. ${ }^{10}$ Recently, a few cases of human ehrlichiosis produced by E. ewingii, transmitted through Amblyomma americanum and Dermacentor variabilis, have been reported. ${ }^{5,11}$

Human ehrlichiosis produces non-specific flulike symptoms such as fever, headache, myalgias, sweats, and malaise similar to rickettsial illnesses. Less common symptoms include nausea, vomiting, anorexia, and confusion. Laboratory findings include leukopenia, thrombocytopenia, and elevated liver enzymes. ${ }^{9,12}$ Although generally a self-limited disease, fatalities have been reported, particularly in immunocompromised patients. ${ }^{13}$

The diagnosis of ehrlichiosis is often made clinically and infections are treated empirically without laboratory evidence of Ehrlichia infection. There are several reasons for this. Ehrlichia do not grow in standard blood culture medium. Serologic indirect fluorescent-antibody tests can be clinically useful in establishing a diagnosis of ehrlichiosis; however, acute-phase serum is often nonreactive and analysis of convalescent serum may be required 
for the diagnosis. ${ }^{14}$ Polymerase chain reaction (PCR) analysis using Ehrlichia-specific primers is another method to detect ehrlichiosis; however, it is often available only at referral centers. Organisms can be identified as punctate deep-blue to gray-blue coccobacilli arranged in spherical groups, or morulae, in the cytoplasm..$^{8,9,15}$ By electron microscopy, the morulae have been shown to be within membranebound cytoplasmic vacuoles where they appear to inhibit the fusion of phagosomes and lysosomes. ${ }^{16,17}$ Peripheral blood smear detection of organisms is theoretically the most rapid way to confirm Ehrlichia infection. Morulae, however, are difficult to identify for inexperienced laboratory personnel and the infected cells can be rare, requiring an extensive search. The purpose of this study was to determine how often ehrlichiosis could be diagnosed by peripheral blood smear and to determine if other peripheral blood smear changes might be predictive of Ehrlichia infection.

\section{Materials and methods}

A total of 23 patients with symptoms and a clinical history consistent with a rickettsial infection who presented to Vanderbilt University Medical Center, Davidson Veterans' Association Medical Center or St Thomas Hospital in Nashville, Tennessee between April and September of 1998 or 1999 were included in this study. Clinical criteria required included a fever $\geq 37.5^{\circ} \mathrm{C}$, a history of tick bite or exposure, and a clinical suspicion of tick-borne rickettsial infection sufficient to warrant initiation of doxycycline therapy. The patient demographics and clinical history including age, sex, rash, central nervous system symptoms, date of presentation, days of illness before presentation, and immune status were recorded. Laboratory data including complete blood count, differential and platelet count and liver function tests were compared using a Wilcoxon W test.

Wright's stained peripheral blood films or buffy coat films were examined for organisms, level of infectivity (\# infected cells/\# white blood cells), and maximum number of morulae per infected cell. In addition, the presence of toxic changes, apoptosis and atypical lymphocytes was graded using the following scheme: '-' absent, ' $+/-$ ' slight, occasional cells with readily identifiable change, ' + ' moderate number of cells with readily identifiable change, and ' ++ ' virtually all cells with readily identifiable change. The entire slide, including at least 500 white blood cells, was examined at high dry magnification with oil-immersion examination when appropriate. White blood cells were examined until organisms were identified or the whole slide was covered. The absolute concentration of morulae-containing cells was calculated by multiplying the absolute number of white blood cells/ $\mu \mathrm{l}$ by the percentage of infected white blood cells counted on peripheral blood film review. Two patients were empirically treated with antibiotics for presumed rickettsial infection for 3-4 days prior to examination of the blood smear. In addition, in three patients serial blood smears were examined following antibiotic therapy.

Culture was performed using a DH82 cell line as previously described ${ }^{18}$ on peripheral blood specimens in 21 cases immediately after collection. DNA extraction and nested PCR of the 16S rRNA gene of general Ehrlichia (species nonspecific), E. chaffeensis, and Anaplasma phagocytophila (the HGE agent), was performed on all peripheral blood samples, as previously described. ${ }^{18,19}$ Both positive and negative control specimens were tested simultaneously with each reaction. The positive control DNA specimens were obtained from culture derived E. chaffeensis and Anaplasma phagocytophila (the HGE agent) (kindly provided by JS Dumler, Johns Hopkins University Hospital). The negative control specimens were sterile water and DNA obtained from the blood of an uninfected person, and uninfected DH82 cells. For patients with PCR evidence of Ehrlichia infection, but with negative PCR results using the species-specific primers, nucleotide sequencing of the 16s rRNA gene was performed using primers $15 \mathrm{~F}$ (ATCATGGCTCAGAACGAACG) and 208R (CGTAGGCTCATCTAATAGCG) to amplify a variable region of the gene useful for species identification. The sequences of these products were compared to the published sequences of the previously known isolates of Ehrlichia. ${ }^{19}$

Cases were considered positive for ehrlichial infection when PCR with the broad range primers was positive and/or the culture was positive.

\section{Results}

In all, 16 (16/23) patients were positive by PCR for ehrlichial infection, including 14 culture positive cases. Of these 15 cases were shown to be E. chaffeensis and one was sequenced as E. ewingii. The cases that were positive by PCR for Ehrlichia had similar clinical characteristics to the PCR negative, culture negative group, which consisted of three cases of Rocky Mountain spotted fever, one case of hepatitis A, and three cases of unknown etiology.

Most laboratory results, including hematocrit and differential counts, were similar between the cases that were positive and negative for ehrlichial infection by PCR analysis, but the mean white blood cell counts and the platelet counts showed a trend toward being lower in the Ehrlichia-infected group (white blood cell count $P=0.07$, standard deviation 6.1; platelet count $P=0.165$, standard deviation 54.3) (Table 1). The Ehrlichia-negative group had higher mean SGOT and SGPT levels, reflecting the exceptionally high levels seen in the 
patient with hepatitis A (SGOT $P=0.9$, standard deviation 658; SGPT $P=0.9$, standard deviation 732).

A review of the peripheral blood films showed more toxic change and atypical lymphocytes in the Ehrlichia-positive group (Table 2). Toxic changes noted included course cytoplasmic azurophilic granulation and vacuolization in granulocytes. The atypical lymphocytes were large with folded, hyperchromatic nuclei, abundant basophilic cytoplasm, and cytoplasmic granules (Figure 1).

Organisms were identified by peripheral blood film review in 6/16 (38\%) Ehrlichia-infected cases (five E. chaffeensis, one E. ewingii), with a sensitivity in E. chaffeensis cases of 33\% (5/15 cases). Cases of $E$. chaffeensis had morulae only in monocytes (Figure 2), whereas the case of $E$. ewingii had morulae predominantly in granulocytes (Figure 3). The morulae were collections of 8-20 punctate, round to slightly angular, deeply blue to gray-blue staining inclusions, usually arranged in a spherelike configuration. The typical rate of infectivity was $<0.2 \%$ of white blood cells; however, four of the six cases with morulae present in the peripheral blood films were immunocompromised, and in two of these patients $1-10 \%$ of white blood cells were infected. Usually, only one morula was seen per infected cell, but occasionally, particularly in immunocompromised patients, numerous morulae could be seen per cell (Table 2, Figure 4). The absolute concentration of morulae-containing cells ranged from $4.2 \mathrm{cells} / \mu \mathrm{l}$ to $1200 \mathrm{cells} / \mu \mathrm{l}$. In two patients who received one to two doses of doxycycline after the initial peripheral smear review, subsequent peripheral blood smears contained occasional morulae within cytoplasmic vacuoles with somewhat pyknotic and hyperchromatic organisms in morulae (Figure 5). Morulae were cleared from the peripheral films within 1-2 days of treatment with doxycycline.

Structures commonly seen and potentially confused with morulae included platelets, Döhle bodies, apoptotic debris, large cytoplasmic granules, and nuclear fragments. In some non-Ehrlichia cases, platelets were seen overlying the cytoplasm of neutrophils, mimicking an Ehrlichia infection, but

Table 1 Laboratory values from patients with clinical symptoms of ehrlichiosis

\begin{tabular}{|c|c|c|c|c|c|}
\hline Normal range & 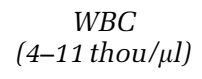 & $\begin{array}{c}P C V \\
(37-50 \%)\end{array}$ & $\begin{array}{c}\text { Platelet count } \\
(150-400 \text { thou/ } \mu \mathrm{l})\end{array}$ & $\begin{array}{c}S G O T \\
(4-40 I U / 1)\end{array}$ & $\begin{array}{c}S G P T \\
(4-40 I U / I)\end{array}$ \\
\hline Ehrlichia positive & 5 & 36 & 69 & 336 & 213 \\
\hline Ehrlichia negative & 8.3 & 36 & 104 & 686 & 936 \\
\hline
\end{tabular}

Table 2 Peripheral blood smear findings from patients with clinical symptoms of ehrlichiosis

\begin{tabular}{|c|c|c|c|c|c|c|c|}
\hline Case & $\begin{array}{l}\text { Ehrlichia } \\
\text { status }\end{array}$ & Morulae & $\begin{array}{c}\# \text { inf cells/ } \\
\text { \#WBC }\end{array}$ & $\begin{array}{c}\text { Max. \# } \\
\text { morulae/cell }\end{array}$ & Toxic change & $\begin{array}{l}\text { Atypical } \\
\text { lymphs }\end{array}$ & Apoptosis \\
\hline 1 & + & Absent & - & NA & + & + & - \\
\hline 2 & + & Absent & - & NA & ++ & ++ & - \\
\hline 3 & + & Present & $<1 / 500$ & 1 & - & + & + \\
\hline 4 & + & Absent & - & NA & \pm & \pm & + \\
\hline 5 & + & Absent & - & NA & - & - & - \\
\hline 6 & + & Absent & - & NA & + & ++ & - \\
\hline 7 & + & Absent & - & NA & + & + & - \\
\hline 8 & + & Present & $<1 / 500$ & 4 & - & + & $\mp$ \\
\hline 9 & + & Present & $<1 / 100$ & $>10$ & ++ & + & + \\
\hline 10 & + & Present & $<1 / 500$ & 1 & ++ & + & ++ \\
\hline 11 & + & Absent & - & - & - & + & - \\
\hline 12 & + & Absent & - & NA & + & ++ & - \\
\hline 13 & + & Present & $<1 / 500$ & 1 & + & + & - \\
\hline 14 & + & Absent & - & NA & $+/++$ & ++ & - \\
\hline 15 & + & Present & $1 / 10$ & 3 & ++ & + & - \\
\hline 16 & - & Absent & - & NA & - & - & - \\
\hline 17 & - & Absent & - & NA & - & - & - \\
\hline 18 & - & Absent & - & NA & - & - & + \\
\hline 19 & - & Absent & - & NA & - & - & - \\
\hline 20 & - & Absent & - & NA & + & $\mp$ & - \\
\hline 21 & - & Absent & - & NA & ++ & - & + \\
\hline 22 & - & Absent & - & NA & + & - & - \\
\hline 23 & - & Absent & - & NA & + & - & - \\
\hline
\end{tabular}

'-', absent; ' \pm ', slight; “+”, moderate; and '++', marked; diagnoses in the Ehrlichia negative group included Rocky Mountain spotted fever in cases 20 and 21, hepatitis $A$ in case 18, and unknown in cases 16, 17, 19, 22, and 23; immunocompromised patients in cases 8, 9, 13 , and 15 . 


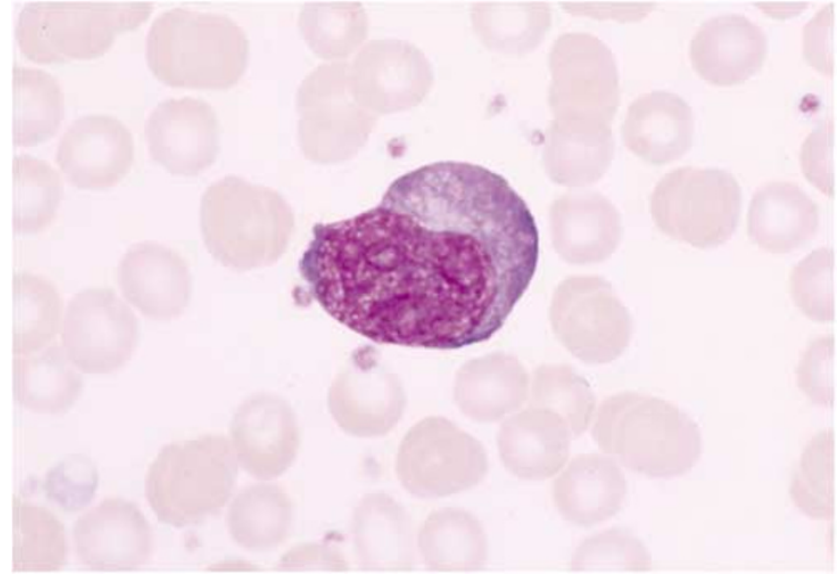

Figure 1 Atypical lymphocytes were more commonly seen in Ehrlichia-infected cases. They were typically large, with hyperchromatic nuclei, abundant basophilic cytoplasm, and contained prominent cytoplasmic granules (Wright's-stained blood film, patient 6 , original magnification $\times 300$ ).



Figure 2 A degenerating monocyte in the feathered edge of the peripheral blood film contains an intracytoplasmic morula of $E$. chaffeensis. The morulae is composed of discreet, punctate grayblue organisms (Wright's-stained blood film, patient 9, original magnification $\times 300$ ).

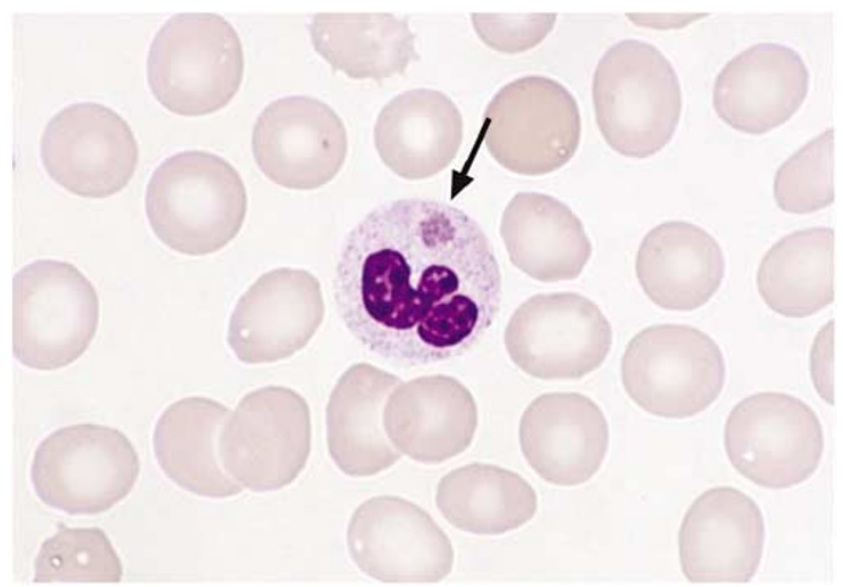

Figure 3 E. ewingii morula, present in a granulocyte, is morphologically indistinguishable from E. chaffeensis (Wright's stained blood film, patient 15 , original magnification $\times 300$ ).

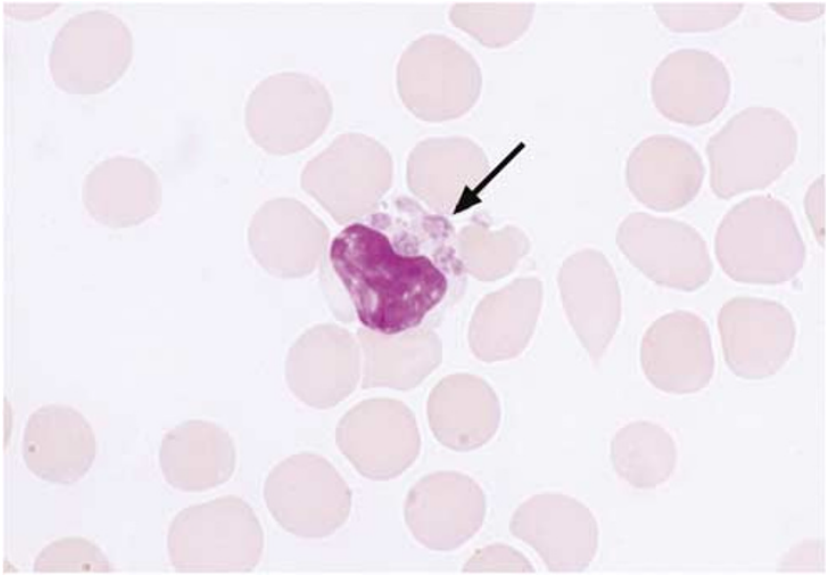

Figure 4 Monocytes from immunocompromised hosts may contain multiple morulae (Wright's stained blood film, patient 9, original magnification $\times 300)$.

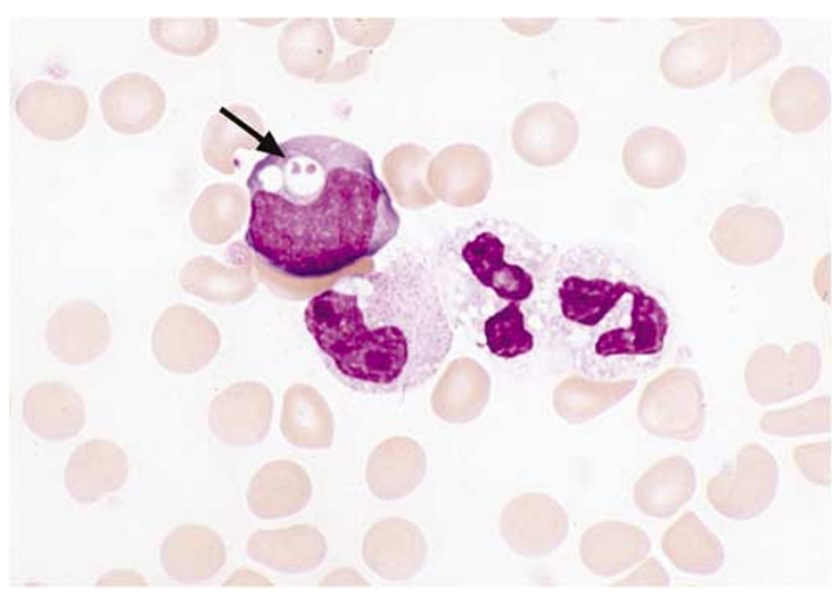

Figure 5 After initiation of doxycycline therapy, organisms rapidly disappear. This monocyte has an intracytoplasmic vacuole containing hyperchromatic and shrunken morula of $E$. chaffeensis after the patient had received two doses of doxycycline (Wright's-stained blood film, patient 10, original magnification $\times 300)$.

platelets were distinguished from morulae by their lack of distinct punctate morphology.

\section{Discussion}

Ehrlichiosis is a potentially fatal disease that is often difficult to diagnose clinically. As acute serologic studies may be negative and many laboratories must send out tests to reference laboratories, it may take weeks to diagnose with serologic or molecular tests or culture. A rapid and inexpensive method to diagnose ehrlichiosis is by identification of morulae on Wright's-stained peripheral blood films. The literature regarding the prevalence of and morphologic descriptions of peripheral blood films containing infected white blood cells in confirmed cases of ehrlichiosis is limited, ${ }^{21}$ so the detection rate and 
associated findings in peripheral blood smears have not been accurately determined. In this study, 38\% of peripheral films from 16 PCR-confirmed cases of ehrlichiosis had morulae in white blood cells. In immunocompromised patients, the level of detection by peripheral blood film examination was $100 \%$, whereas in immunocompetent patients, the level of detection was only $17 \%$. This increased sensitivity is important as the immunocompromised group of patients is at increased risk for rapidly fatal ehrlichial infections. ${ }^{13,20,21}$ Future studies to confirm these findings with larger cohorts would be valuable.

Significant changes were quickly seen in the morulae following treatment with doxycycline, including development of a visible cytoplasmic vacuole surrounding organisms that appeared more hyperchromatic and shrunken. These changes were seen after one to two doses of antibiotic therapy. After 2 days of therapy, organisms were no longer identifiable on peripheral blood films, making it unlikely that organisms can be identified if empiric therapy has been given for 2 days or more.

Finally, one of the most relevant findings in our study was the presence of marked toxic change and prominent large granular lymphocytes in cases of ehrlichiosis. These large granular lymphocytes likely represent the increased $\gamma \delta \mathrm{T}$ cells identified previously by flow cytometry. ${ }^{22}$ On review of the peripheral blood film, the large granular lymphocytes often had very atypical, folded, hyperchromatic nuclei, and cytoplasmic granules, and could be confused with neoplastic NK or NK-like T cells. Toxic changes and apoptosis were more pronounced in the cases that were proven to be infected by Ehrlichia, but could be seen in some non-Ehrlichia cases. The finding of atypical large granular lymphocytes and moderate to marked toxic change is unusual in other infections. Although not in of itself diagnostic, these peripheral blood smear findings should suggest the possibility of Ehrlichia infection and prompt a careful review of the peripheral blood films for characteristic morulae, as well as serologic testing and PCR analysis when morulae are not identified.

In conclusion, a systematic and thorough review of Wright's-stained peripheral films is a specific and relatively sensitive method for diagnosing ehrlichiosis, particularly in immunocompromised patients with a history of tick bite or possible tick exposure. Ehrlichiosis should be suspected when a patient has leukopenia, thrombocytopenia, and toxic changes, apoptosis, and large granular lymphocytes in Wright's stained peripheral blood films and a history of tick bite or possible tick exposure. Shortly after initiation of antibiotic therapy, degenerated of morulae within cytoplasmic vacuoles are present, and organisms are not detected morphologically after 2 days of therapy.

\section{References}

1 Cowdry EV. Studies on the aetiology of heartwater. I. Observation of a rickettsia, Rickettsia ruminantium (n. sp.) in the tissues of infected animals. J Exp Med 1925;44:803-814.

2 Donatien A, Lestoquard F. Existence en Algerie d'une Rickettsia du chein. Bull Pathol Exot 1935;28:418-419.

3 Dawson JE. Human ehrlichiosis in the United States. Curr Clin Top Infect Dis 1996;16:164-171.

4 Perez M, Rikihisa Y, Wen B. Ehrlichia canis-like agent isolated from a man in Venezuela: antigenic and genetic characterization. J Clin Microbiol 1996;34: 2133-2139.

5 Buller RS, Arens M, Hmiel SP, et al. Ehrlichia ewingii, a newly recognized agent of human ehrlichiosis. N Engl J Med 1999;341:148-155.

6 Ewing SA, Dawson JE, Kocan AA, et al. Experimental transmission of Ehrlichia chaffeensis (Rickettseales: Ehrlichieae) among white-tailed deer by Amblyomma americanum (Acari: Ixodidae). J Med Entomol 1995;32:368-374.

7 Anderson BE, Dawson JE, Jones DC, et al. Ehrlichia chaffeensis, a new species associated with human ehrlichiosis. J Clin Microbiol 1991;29:2838-2842.

8 Bakken JS, Dumler JS, Chen SM, et al. Human granulocytic erhlichiosis in the upper midwest United States: a new species emerging? J Am Med Assoc 1994;272:212-218.

9 Bakken JS, Krueth J, Wilson-Nordskog C, et al. Clinical and laboratory characteristics of human granulocytic ehrlichiosis. J Am Med Assoc 1996;275: 199-205

10 Dumler JS, Barbet AF, Bekker CP, et al. Reorganization of genera in the families Rickettsiaceae and Anaplasmataceae in the order Rickettsiales: unification of some species of Ehrlichia and Anaplasma, Cowdria with Ehrlichia and Ehrlichia with Neorickettsia, descriptions of six new species combinations and designation of Ehrlichia equi and "HGE agent" as subjective synonyms of Ehrlichia phagocytophila. Int J Syst Bacteriol 2001;51:2145-2165.

11 Anziani OS, Ewing SA, Barker RW. Experimental transmission of a granulocytic form of the tribe Ehrlichieae by Dermacentor variabilis and Amblyomma americanum to dogs. Am J Vet Res 1990;51:929-931.

12 Fishbein DB, Dawson JE, Robinson LE. Human ehrlichiosis in the United States, 1985 to 1990. Ann Intern Med 1994;120:736-743.

13 Paddock CD, Suchard DP, Grumbach KL, et al. Brief report: fatal seronegative ehrlichiosis in a patient with HIV infection. N Engl J Med 1993;329: 1164-1167.

14 Aguero-Rosenfeld ME, Kalantarpour F, Balach M, et al. Serology of culture-confirmed cases of human granulocytic ehrlichiosis. J Clin Microbiol 2000;38:635-638.

15 Dumler JS, Dawson JE, Walker DH. Human ehrlichiosis: hematopathology and immunohistiologic detection of Ehrlichia chaffeensis. Hum Pathol 1993;24: 391-396.

16 Webster P, Ijdo JW, Chicoine LM, et al. The agent of human granulocytic ehrlichiosis resides in an endosomal compartment. J Clin Invest 1998;101: 1932-1941.

17 Barnewall RE, Rikihisa Y, Lee EH. Ehrlichia chaffeensis inclusion are early endosomes which selectively 
accumulate transferrin receptor. Infect Immun 1997;65:1455-1461.

18 Standaert SM, Yu T, Scott MA, et al. Primary isolation of Ehrlichia chaffeensis from patients with febrile illnesses: clinical and molecular characteristics. J Inf Dis 2000;181:1082-1088.

19 Warner CK, Dawson JE. Genus- and species-level identification of Ehrlichia species by PCR and sequencing. In: Pershing DE, (ed). PCR Protocols for Emerging Infectious Diseases. ASM Press: Washington, DC, 1996, pp. 100-105.
20 Telford SR, Lepore TJ, Snow P, et al. Human granulocytic ehrlichiosis in Massachusetts. Ann Int Med 1995;123:277-279.

21 Paddock CD, Folk SM, Shore GM, et al. Infections with Ehrlichia chaffeensis and Ehrlichia ewingii in persons coinfected with human immunodeficiency virus. Clin Infect Dis 2001;33: 1586-1594.

22 Caldwell CW, Everett ED, McDonald G. Apoptosis of $\gamma /$ $\delta \mathrm{T}$ cells in human ehrlichiosis. Am J Clin Pathol 1996;105:640-646. 